\title{
Integer Powers of Certain Complex Pentadiagonal 2-Toeplitz Matrices
}

\author{
Hatice Kübra Duru*and Durmuş Bozkurt ${ }^{\dagger}$ \\ Selcuk University, Science Faculty Department of Mathematics, \\ Turkey
}

July 5, 2018

\begin{abstract}
In this study, we get a general expression for the entries of the sth power of even order pentadiagonal 2-Toeplitz matrices.
\end{abstract}

\section{Introduction}

Gover 11 gained eigenvalues and eigenvectors of a tridiagonal 2-Toeplitz matrix in terms of the Chebyshev polynomials. Hadj and Elouafi [2] obtained the general expression of the characteristic polynomial, determinant and eigenvectors for pentadiagonal matrices. Rimas [3] offered general expression for the entries of the power of tridiagonal 2-Toeplitz matrix, in terms of the Chebyshev polynomials of the second kind. Obvious formulas for the determinants of a band symmetric Toeplitz matrix is restated in [4. Álvarez-Nodarse et al. [5] gained the general expressions for the eigenvalues, eigenvectors and the spectral measure of 2 and 3-Toeplitz matrices. The powers of even order symmetric pentadiagonal matrices are calculated in [6]. Öteleş and Akbulak [7] viewed powers of tridiagonal matrices. $\mathrm{Wu}[\mathrm{8}$ calculated the powers of Toeplitz Matrices. The powers of complex pentadiagonal Toeplitz matrices are computed in $[9]$.

This paper is organized as follows: the first section, motivated by [2, we apply $K_{n}$ pentadiagonal 2-Toeplitz matrix to the characteristic polynomial and eigenvectors of this matrix in given [2. In Section 2, we obtain the eigenvalues and eigenvectors of $K_{n}$ pentadiagonal 2-Toeplitz matrix. In Section 3, the $s$ th power of pentadiagonal 2-Toeplitz matrix we will get by using the expression $K_{n}^{s}=L_{n} J_{n}^{s} L_{n}^{-1}$ [11], where $J_{n}$ is the Jordan's form of $K_{n}$ and $L_{n}$ is the transforming matrix. In Section 4 , some numerical examples are given.

Consider the polynomial sequence $\left\{A_{i}\right\}_{i \geq 0}$ and $\left\{B_{i}\right\}_{i \geq 0}$ characterized by a three-term recurrence relation

\footnotetext{
*hkduru@selcuk.edu.tr

${ }^{\dagger}$ dbozkurt@selcuk.edu.tr
} 


$$
\begin{aligned}
& x A_{0}(x)=a_{1} A_{0}(x)+b_{1} A_{2}(x) \\
& x A_{1}(x)=a_{2} A_{1}(x)+b_{2} A_{3}(x) \\
& x A_{i-1}(x)=c_{1} A_{i-3}(x)+a_{1} A_{i-1}(x)+b_{1} A_{i+1}(x) \text { for } i \geq 3 \text { and } i=2 t+1(t \in \mathbb{N}) \\
& x A_{i-1}(x)=c_{2} A_{i-3}(x)+a_{2} A_{i-1}(x)+b_{2} A_{i+1}(x) \text { for } i \geq 3 \text { and } i=2 t(t \in \mathbb{N})
\end{aligned}
$$

with initial conditions $A_{0}(x)=0$ and $A_{1}(x)=1$, and

$$
\begin{aligned}
& x B_{0}(x)=a_{1} B_{0}(x)+b_{1} B_{2}(x) \\
& x B_{1}(x)=a_{2} B_{1}(x)+b_{2} B_{3}(x) \\
& x B_{i-1}(x)=c_{1} B_{i-3}(x)+a_{1} B_{i-1}(x)+b_{1} B_{i+1}(x) \text { for } i \geq 3 \text { and } i=2 t+1(t \in \mathbb{N}) \\
& x B_{i-1}(x)=c_{2} B_{i-3}(x)+a_{2} B_{i-1}(x)+b_{2} B_{i+1}(x) \text { for } i \geq 3 \text { and } i=2 t(t \in \mathbb{N})
\end{aligned}
$$

with initial conditions $B_{0}(x)=1$ and $B_{1}(x)=0$, here $a_{1}, a_{2} \in \mathbb{C}$ and $b_{1}, b_{2}, c_{1}, c_{2} \in$ $\mathbb{C} \backslash\{0\}$. We can write a matrix form to this three-term recurrence relations

$$
\begin{aligned}
& x A_{n-1}(x)=K_{n} A_{n-1}(x)+A_{n}(x) d_{n-1}+A_{n+1}(x) d_{n} \\
& x B_{n-1}(x)=K_{n} B_{n-1}(x)+B_{n}(x) d_{n-1}+B_{n+1}(x) d_{n}
\end{aligned}
$$

where $A_{n-1}(x)=\left[A_{0}(x), A_{1}(x), A_{2}(x), \ldots, A_{n-1}(x)\right]^{T}$, $B_{n-1}(x)=\left[B_{0}(x), B_{1}(x), B_{2}(x), \ldots, B_{n-1}(x)\right]^{T}$,

$$
\left.\begin{array}{l}
d_{n-1}=\left[0,0,0, \ldots, 0, b_{2}, 0\right]^{T} \\
d_{n}=\left[0,0,0, \ldots, 0,0, b_{1}\right]^{T}
\end{array}\right\}(n=2 t+1, t \in \mathbb{N})
$$

and

$$
\left.\begin{array}{l}
d_{n-1}=\left[0,0,0, \ldots, 0, b_{1}, 0\right]^{T} \\
d_{n}=\left[0,0,0, \ldots, 0,0, b_{2}\right]^{T}
\end{array}\right\}(n=2 t, t \in \mathbb{N})
$$

Let

$$
K_{n}=\left[\begin{array}{cccccccccc}
a_{1} & 0 & b_{1} & 0 & 0 & 0 & \cdots & 0 & 0 & 0 \\
0 & a_{2} & 0 & b_{2} & 0 & 0 & \cdots & 0 & 0 & 0 \\
c_{1} & 0 & a_{1} & 0 & b_{1} & 0 & \cdots & 0 & 0 & 0 \\
0 & c_{2} & 0 & a_{2} & 0 & b_{2} & \cdots & 0 & 0 & 0 \\
0 & 0 & c_{1} & 0 & a_{1} & 0 & \cdots & 0 & 0 & 0 \\
\vdots & \vdots & \vdots & \vdots & \vdots & \vdots & \ddots & \vdots & \vdots & \vdots \\
0 & 0 & 0 & 0 & 0 & 0 & \cdots & 0 & b_{2} & 0 \\
0 & 0 & 0 & 0 & 0 & 0 & \cdots & a_{1} & 0 & b_{1} \\
0 & 0 & 0 & 0 & 0 & 0 & \cdots & 0 & a_{2} & 0 \\
0 & 0 & 0 & 0 & 0 & 0 & \cdots & c_{1} & 0 & a_{1} \\
& & & & & & & & &
\end{array}\right] \text { for } n=2 t+1(t \in \mathbb{N})
$$


and

$$
K_{n}=\left[\begin{array}{cccccccccc}
a_{1} & 0 & b_{1} & 0 & 0 & 0 & \cdots & 0 & 0 & 0 \\
0 & a_{2} & 0 & b_{2} & 0 & 0 & \cdots & 0 & 0 & 0 \\
c_{1} & 0 & a_{1} & 0 & b_{1} & 0 & \cdots & 0 & 0 & 0 \\
0 & c_{2} & 0 & a_{2} & 0 & b_{2} & \cdots & 0 & 0 & 0 \\
0 & 0 & c_{1} & 0 & a_{1} & 0 & \cdots & 0 & 0 & 0 \\
\vdots & \vdots & \vdots & \vdots & \vdots & \vdots & \ddots & \vdots & \vdots & \vdots \\
0 & 0 & 0 & 0 & 0 & 0 & \cdots & 0 & b_{1} & 0 \\
0 & 0 & 0 & 0 & 0 & 0 & \cdots & a_{2} & 0 & b_{2} \\
0 & 0 & 0 & 0 & 0 & 0 & \cdots & 0 & a_{1} & 0 \\
0 & 0 & 0 & 0 & 0 & 0 & \cdots & c_{2} & 0 & a_{2} \\
& & & & & & & & &
\end{array}\right] \text { for } n=2 t(t \in \mathbb{N}) \quad(5)
$$

here $a_{1}, a_{2} \in \mathbb{C}$ and $b_{1}, b_{2}, c_{1}, c_{2} \in \mathbb{C} \backslash\{0\}$.

Lemma 1 The polynomial sequences $\left\{A_{i}\right\}_{i \geq 0}$ and $\left\{B_{i}\right\}_{i \geq 0}$ confirm

$$
\begin{aligned}
& \operatorname{deg}\left(A_{2 i+1}\right)=i \text { and the leading coefficient of } A_{2 i+1} \text { is equal to } \frac{1}{b_{2}^{i}} \\
& \operatorname{deg}\left(A_{2 i}\right)=0 \\
& \operatorname{deg}\left(B_{2 i+1}\right)=0 \\
& \operatorname{deg}\left(B_{2 i}\right)=i \text { and the leading coefficient of } B_{2 i} \text { is equal to } \frac{1}{b_{1}^{2}} .
\end{aligned}
$$

Proof. Let us prove by the inductive method. For the basis step, we possess

For $i=0: A_{0}(x)=0, B_{0}(x)=1$.

For $i=1: A_{1}(x)=1, B_{1}(x)=0$.

For $i=2: A_{2}(x)=0, B_{2}(x)=\frac{x-a_{1}}{b_{1}}$.

For $i=3: A_{3}(x)=\frac{x-a_{2}}{b_{2}}, B_{3}(x)=0$.

For $i=4: A_{4}(x)=0, B_{4}(x)=\frac{\left(x-a_{1}\right)^{2}-b_{1} c_{1}}{b_{1}^{2}}$.

Assuming that (11) and (2) are correct for $p=k \geq 3$. We will prove it for $k=p+1$, we obtain

$$
\begin{aligned}
& x A_{p-1}(x)=c_{1} A_{p-3}(x)+a_{1} A_{p-1}(x)+b_{1} A_{p+1}(x) \text { for } p \geq 3 \text { and } p=2 t+1(t \in \mathbb{N}) \\
& x A_{p-1}(x)=c_{2} A_{p-3}(x)+a_{2} A_{p-1}(x)+b_{2} A_{p+1}(x) \text { for } p \geq 3 \text { and } p=2 t(t \in \mathbb{N}) \\
& x B_{p-1}(x)=c_{1} B_{p-3}(x)+a_{1} B_{p-1}(x)+b_{1} B_{p+1}(x) \text { for } p \geq 3 \text { and } p=2 t+1(t \in \mathbb{N}) \\
& x B_{p-1}(x)=c_{2} B_{p-3}(x)+a_{2} B_{p-1}(x)+b_{2} B_{p+1}(x) \text { for } p \geq 3 \text { and } p=2 t(t \in \mathbb{N}) .
\end{aligned}
$$

If $p:=2 i$, then we have

$$
\begin{aligned}
& x A_{2 i-1}(x)=c_{2} A_{2 i-3}(x)+a_{2} A_{2 i-1}(x)+b_{2} A_{2 i+1}(x) \\
& x B_{2 i-1}(x)=c_{2} B_{2 i-3}(x)+a_{2} B_{2 i-1}(x)+b_{2} B_{2 i+1}(x) .
\end{aligned}
$$

Accordingly

$$
\begin{aligned}
\operatorname{deg}\left(A_{2 i+1}(x)\right) & =\operatorname{deg}\left(x A_{2 i-1}(x)\right) \\
\operatorname{deg}\left(B_{2 i+1}(x)\right) & =0
\end{aligned}
$$


and the leading coefficient of

$$
\begin{aligned}
A_{2 i+1} & \left.=\frac{1}{b_{2}} \text { (leading coefficient of } A_{2 i-1}(x)\right), \\
& =\frac{1}{b_{2}} \frac{1}{\left(b_{2}\right)^{i-1}}=\frac{1}{\left(b_{2}\right)^{2}} .
\end{aligned}
$$

If $p:=2 i-1$, then we write

$$
\begin{aligned}
& x A_{2 i-2}(x)=c_{1} A_{2 i-4}(x)+a_{1} A_{2 i-2}(x)+b_{1} A_{2 i}(x) \\
& x B_{2 i-2}(x)=c_{1} B_{2 i-4}(x)+a_{1} B_{2 i-2}(x)+b_{1} B_{2 i}(x) .
\end{aligned}
$$

So,

$$
\begin{aligned}
\operatorname{deg}\left(A_{2 i}(x)\right) & =0 \\
\operatorname{deg}\left(B_{2 i}(x)\right) & =\operatorname{deg}\left(x B_{2 i-2}(x)\right)
\end{aligned}
$$

and the leading coefficient of

$$
\begin{aligned}
B_{2 i} & =\frac{1}{b_{1}}\left(\text { leading coefficient of } B_{2 i-2}(x)\right), \\
& =\frac{1}{b_{1}} \frac{1}{\left(b_{1}\right)^{i-1}}=\frac{1}{\left(b_{1}\right)^{2}} .
\end{aligned}
$$

Definition $2 K_{n}$ be $n$-square pentadiagonal 2-Toeplitz matrix, one correlates the sequence polynomial $P_{i}$ described by

$$
P_{i}=\operatorname{det}\left[\begin{array}{cc}
A_{n} & A_{i} \\
B_{n} & B_{i}
\end{array}\right] .
$$

Lemma 3 Due to (1) and (2), we own

$$
\begin{aligned}
x P_{n-1}(x) & =K_{n} P_{n-1}(x)+P_{n}(x) d_{n-1}+P_{n+1}(x) d_{n} \\
& =K_{n} P_{n-1}(x)+P_{n+1}(x) d_{n}
\end{aligned}
$$

where $P_{n-1}(x)=\left[P_{0}(x), P_{1}(x), P_{2}(x), \ldots, P_{n-1}(x)\right]^{T}$,

$$
\left.\begin{array}{rl}
d_{n-1}= & {\left[0,0,0, \ldots, 0, b_{2}, 0\right]^{T}} \\
d_{n} & =\left[0,0,0, \ldots, 0,0, b_{1}\right]^{T}
\end{array}\right\}(n=2 t+1, t \in \mathbb{N}),
$$

Lemma 4 The polynomial $P_{n+1}$ is degree $n$ and the leading coefficient of $P_{n+1}$ is

$$
\begin{array}{ll}
-\frac{1}{\left(b_{1} b_{2}\right)^{i}} \quad, \quad \text { if } n=2 t(t \in \mathbb{N}) \\
\frac{1}{b_{1}^{i+1} b_{2}^{i}} \quad, \quad \text { if } n=2 t+1(t \in \mathbb{N}) .
\end{array}
$$


Proof. If $n=2 i$

$$
P_{2 i+1}=\operatorname{det}\left[\begin{array}{ll}
A_{2 i} & A_{2 i+1} \\
B_{2 i} & B_{2 i+1}
\end{array}\right]=A_{2 i} B_{2 i+1}-A_{2 i+1} B_{2 i}
$$

and using Lemma 1

$$
\operatorname{deg}\left(P_{2 i+1}\right)=\operatorname{deg}\left(A_{2 i+1} B_{2 i}\right)
$$

and the leading coefficient of

$$
P_{2 i+1}=-\frac{1}{b_{2}^{i}} \frac{1}{b_{1}^{i}}=-\frac{1}{\left(b_{1} b_{2}\right)^{i}} .
$$

$$
\begin{aligned}
& \text { If } n=2 i+1 \\
& \qquad P_{2 i+1}=\operatorname{det}\left[\begin{array}{ll}
A_{2 i+1} & A_{2 i+2} \\
B_{2 i+1} & B_{2 i+2}
\end{array}\right]=A_{2 i+1} B_{2 i+2}-A_{2 i+2} B_{2 i+1}
\end{aligned}
$$

and using Lemma 1

$$
\operatorname{deg}\left(P_{2 i+2}\right)=\operatorname{deg}\left(A_{2 i+1} B_{2 i+2}\right)
$$

and the leading coefficient of

$$
P_{2 i+2}=\frac{1}{b_{2}^{i}} \frac{1}{b_{1}^{i+1}}
$$

Lemma 5 If $\alpha$ is a zero of the polynomial $P_{n+1}$ then $\alpha$ is an eigenvalues of the matrix $K_{n}$.

Proof. Let $\alpha$ is a zero of the polynomial $P_{n+1}$, from equation (10), we acquire

$$
K_{n} P_{n-1}(\alpha)=\alpha P_{n-1}(\alpha) .
$$

There are four cases to be noted.

Case I. Suppose for $n=2 t(t \in \mathbb{N})$ either $A_{n+1}(\alpha) \neq 0$ or $B_{n}(\alpha) \neq 0$. In that case $P_{0}(\alpha)=A_{n+1}(\alpha)$ and $P_{1}(\alpha)=-B_{n}(\alpha)$, then $P_{n-1}(\alpha)$ is a corresponding non-null eigenvector of $K_{n}$, we acquire that $\alpha$ is an eigenvalue of the matrix $K_{n}$.

Case II. Suppose for $n=2 t-1(t \in \mathbb{N})$ either $A_{n}(\alpha) \neq 0$ or $B_{n+1}(\alpha) \neq 0$. In that case $P_{0}(\alpha)=A_{n}(\alpha)$ and $P_{1}(\alpha)=-B_{n+1}(\alpha)$, then $P_{n-1}(\alpha)$ is a corresponding non-null eigenvector of $K_{n}$, we acquire that $\alpha$ is an eigenvalue of the matrix $K_{n}$.

Case III. Suppose for $n=2 t(t \in \mathbb{N}), A_{n}(\alpha)=B_{n+1}(\alpha)=0$ and $B_{n}(\alpha) \neq$ 0 . Let $F_{n-1}(\alpha)=-A_{n-1}(\alpha) B_{n}(\alpha)$, we own $K_{n} F_{n-1}(\alpha)=\alpha F_{n-1}(\alpha)$, then $F_{n-1}(\alpha)$ is a corresponding non-null eigenvector of $K_{n}$, we acquire that $\alpha$ is an 
eigenvalue of the matrix $K_{n}$.

Case IV. Suppose for $n=2 t+1(t \in \mathbb{N}), A_{n-1}(\alpha)=B_{n}(\alpha)=0$ and $A_{n}(\alpha) \neq 0$. Let $F_{n-1}(\alpha)=A_{n}(\alpha) B_{n-1}(\alpha)$, we own $K_{n} F_{n-1}(\alpha)=\alpha F_{n-1}(\alpha)$, then $F_{n-1}(\alpha)$ is a corresponding non-null eigenvector of $K_{n}$, we acquire that $\alpha$ is an eigenvalue of the matrix $K_{n}$.

Theorem 6 Let $K_{n}$ be n-square pentadiagonal 2-Toeplitz matrix and the corresponding polynomial $P_{n+1}$ in the eq. (10). Suppose that $P_{n+1}$ has simple zeros, the characteristic polynomial of $K_{n}$ is completely

$$
\begin{array}{ll}
\left|x I_{n}-K_{n}\right|=\left(b_{1} b_{2}\right)^{\frac{n}{2}} A_{n+1} B_{n}, & \text { if } n=2 t(t \in \mathbb{N}) \\
\left|x I_{n}-K_{n}\right|=b_{1}^{\frac{n+1}{2}} b_{2}^{\frac{n-1}{2}} A_{n} B_{n+1}, & \text { if } n=2 t+1(t \in \mathbb{N})
\end{array}
$$

here $I_{n}$ is the $n$-square identity matrix.

Proof. Let $\alpha_{1}, \ldots, \alpha_{n}$ the zeros of characteristic polynomial of $K_{n}$. Lemma $5, \alpha_{1}, \ldots, \alpha_{n}$ are the eigenvalues of the matrix $K_{n}$.

\section{Eigenvalues and eigenvectors of $K_{n}$}

Theorem 7 Let $K_{n}$ be $n$-square $(n=2 t, t \in \mathbb{N})$ pentadiagonal 2-Toeplitz matrix as in (5). Then the eigenvalues and eigenvectors of the matrix $K_{n}$ are

$$
\alpha_{k}= \begin{cases}a_{1}-2 \sqrt{b_{1} c_{1}} \cos \left(\frac{(k+1) \pi}{n+2}\right), & (k=2 t+1, t \in \mathbb{N}) \\ a_{2}-2 \sqrt{b_{2} c_{2}} \cos \left(\frac{k \pi}{n+2}\right), & (k=2 t, t \in \mathbb{N})\end{cases}
$$

and

$$
\left[\begin{array}{c}
B_{0}\left(\alpha_{j}\right) \\
B_{1}\left(\alpha_{j}\right) \\
B_{2}\left(\alpha_{j}\right) \\
\vdots \\
B_{n-2}\left(\alpha_{j}\right) \\
B_{n-1}\left(\alpha_{j}\right)
\end{array}\right] \quad(j=1,3,5, \ldots, n-3, n-1)
$$

and

$$
\left[\begin{array}{c}
A_{0}\left(\alpha_{j}\right) \\
A_{1}\left(\alpha_{j}\right) \\
A_{2}\left(\alpha_{j}\right) \\
\vdots \\
A_{n-2}\left(\alpha_{j}\right) \\
A_{n-1}\left(\alpha_{j}\right)
\end{array}\right] \quad(j=2,4,6, \ldots, n-2, n)
$$


Proof. We obtain for

$$
b_{2}^{\frac{n}{2}} A_{n+1}(x)=\left(b_{2} c_{2}\right)^{\frac{n}{4}} U_{\frac{n}{2}}\left(\frac{x-a_{2}}{2 \sqrt{b_{2} c_{2}}}\right)
$$

and

$$
b_{1}^{\frac{n}{2}} B_{n}(x)=\left(b_{1} c_{1}\right)^{\frac{n}{4}} U_{\frac{n}{2}}\left(\frac{x-a_{1}}{2 \sqrt{b_{1} c_{1}}}\right)
$$

from the recurrence relations (11) and (2), here $n=2 t(t \in \mathbb{N})$ and $U_{n}($.$) is the$ $n$th degree Chebyshev polynomial of the second kind [10]:

$$
U_{n}(x)=\frac{\sin ((n+1) \arccos x)}{\sin (\arccos x)}
$$

All the roots of $U_{n}(x)$ are included in the interval $[-1,1]$. Due to (12), (16) and (17), we have

$$
\begin{aligned}
\left|x I_{n}-K_{n}\right| & =\left(b_{1} c_{1}\right)^{\frac{n}{4}} U_{\frac{n}{2}}\left(\frac{x-a_{1}}{2 \sqrt{b_{1} c_{1}}}\right)\left(b_{2} c_{2}\right)^{\frac{n}{4}} U_{\frac{n}{2}}\left(\frac{x-a_{2}}{2 \sqrt{b_{2} c_{2}}}\right) \\
& =\left(b_{1} c_{1} b_{2} c_{2}\right)^{\frac{n}{4}} U_{\frac{n}{2}}\left(\frac{x-a_{1}}{2 \sqrt{b_{1} c_{1}}}\right) U_{\frac{n}{2}}\left(\frac{x-a_{2}}{2 \sqrt{b_{2} c_{2}}}\right) .
\end{aligned}
$$

The eigenvalues of $K_{n}$ obtained as

$$
\alpha_{k}= \begin{cases}a_{1}-2 \sqrt{b_{1} c_{1}} \cos \left(\frac{(k+1) \pi}{n+2}\right), & (k=2 t+1, t \in \mathbb{N}) \\ a_{2}-2 \sqrt{b_{2} c_{2}} \cos \left(\frac{k \pi}{n+2}\right), & (k=2 t, t \in \mathbb{N})\end{cases}
$$

from (18). In 2 Hadj and Elouafi proved eigenvectors of a pentadiagonal matrix. Based on Theorem 6 and [2], we can calculate eigenvectors of the matrix $K_{n}$

\section{The integer powers of the matrix $K_{n}$}

Considering (14) and (15), we write down the transforming matrices $L_{n}$ $(n=2 t, t \in \mathbb{N})$ as following:

$$
L_{n}=\left[\begin{array}{cccc}
B_{0}\left(\alpha_{1}\right) & A_{0}\left(\alpha_{2}\right) & B_{0}\left(\alpha_{3}\right) & A_{0}\left(\alpha_{4}\right) \\
B_{1}\left(\alpha_{1}\right) & A_{1}\left(\alpha_{2}\right) & B_{1}\left(\alpha_{3}\right) & A_{1}\left(\alpha_{4}\right) \\
B_{2}\left(\alpha_{1}\right) & A_{2}\left(\alpha_{2}\right) & B_{2}\left(\alpha_{3}\right) & A_{2}\left(\alpha_{4}\right) \\
\vdots & \vdots & \vdots & \vdots \\
B_{n-3}\left(\alpha_{1}\right) & A_{n-3}\left(\alpha_{2}\right) & B_{n-3}\left(\alpha_{3}\right) & A_{n-3}\left(\alpha_{4}\right) \\
B_{n-2}\left(\alpha_{1}\right) & A_{n-2}\left(\alpha_{2}\right) & B_{n-2}\left(\alpha_{3}\right) & A_{n-2}\left(\alpha_{4}\right) \\
B_{n-1}\left(\alpha_{1}\right) & A_{n-1}\left(\alpha_{2}\right) & B_{n-1}\left(\alpha_{3}\right) & A_{n-1}\left(\alpha_{4}\right)
\end{array}\right.
$$




$$
\begin{array}{ccc}
\cdots & B_{0}\left(\alpha_{n-1}\right) & A_{0}\left(\alpha_{n}\right) \\
\cdots & B_{1}\left(\alpha_{n-1}\right) & A_{1}\left(\alpha_{n}\right) \\
\cdots & B_{2}\left(\alpha_{n-1}\right) & A_{2}\left(\alpha_{n}\right) \\
\ddots & \vdots & \vdots \\
\cdots & B_{n-3}\left(\alpha_{n-1}\right) & A_{n-3}\left(\alpha_{n}\right) \\
\cdots & B_{n-2}\left(\alpha_{n-1}\right) & A_{n-2}\left(\alpha_{n}\right) \\
\cdots & B_{n-1}\left(\alpha_{n-1}\right) & A_{n-1}\left(\alpha_{n}\right)
\end{array}
$$

Now, let us find the inverse matrix $L_{n}^{-1}$ of the matrix $L_{n}$. If we denote $i$-th row of the inverse matrix $L_{n}^{-1}$ by $\mu_{i}$, then we obtain

$$
\left[\begin{array}{c}
q_{i} r_{1}^{l} B_{0}\left(\alpha_{i}\right) \\
q_{i} r_{1}^{l} B_{1}\left(\alpha_{i}\right) \\
q_{i} r_{1}^{l} B_{2}\left(\alpha_{i}\right) \\
q_{i} r_{1}^{l} B_{3}\left(\alpha_{i}\right) \\
q_{i} r_{1}^{l} B_{4}\left(\alpha_{i}\right) \\
\vdots \\
q_{i} r_{1}^{l} B_{n-4}\left(\alpha_{i}\right) \\
q_{i} r_{1}^{l} B_{n-3}\left(\alpha_{i}\right) \\
q_{i} r_{1}^{l} B_{n-2}\left(\alpha_{i}\right) \\
q_{i} r_{1}^{l} B_{n-1}\left(\alpha_{i}\right)
\end{array}\right]^{T} \quad(i=1,3,5, \ldots, n-3, n-1)
$$

and

$$
\left[\begin{array}{c}
q_{i} r_{2}^{l} A_{0}\left(\alpha_{i}\right) \\
q_{i} r_{2}^{l} A_{1}\left(\alpha_{i}\right) \\
q_{i} r_{2}^{l} A_{2}\left(\alpha_{i}\right) \\
q_{i} r_{2}^{l} A_{3}\left(\alpha_{i}\right) \\
q_{i} r_{2}^{l} A_{4}\left(\alpha_{i}\right) \\
\vdots \\
q_{i} r_{2}^{l} A_{n-4}\left(\alpha_{i}\right) \\
q_{i} r_{2}^{l} A_{n-3}\left(\alpha_{i}\right) \\
q_{i} r_{2}^{l} A_{n-2}\left(\alpha_{i}\right) \\
q_{i} r_{2}^{l} A_{n-1}\left(\alpha_{i}\right)
\end{array}\right]^{T} \quad(i=2,4,6, \ldots, n-2, n)
$$

where $r_{1}=\sqrt{\frac{b_{1}}{c_{1}}}, r_{2}=\sqrt{\frac{b_{2}}{c_{2}}}$,

$$
l= \begin{cases}j-1, & j=1,3,5, \ldots, n-3, n-1 \\ j-2, & j=2,4,6, \ldots, n-2, n\end{cases}
$$

and

$$
q_{i}= \begin{cases}\frac{4-\left(\frac{\alpha_{i}-a_{1}}{\sqrt{b_{1} c_{1}}}\right)^{2}}{n+2}, & i=2 t+1(t \in \mathbb{N}) \\ \frac{4-\left(\frac{\alpha_{i}-a_{2}}{\sqrt{b_{2} c_{2}}}\right)^{2}}{n+2}, & i=2 t(t \in \mathbb{N}) .\end{cases}
$$


Thus, we obtain

$$
L_{n}^{-1}=\left[\begin{array}{cccc}
q_{1} B_{0}\left(\alpha_{1}\right) & q_{1} B_{1}\left(\alpha_{1}\right) & q_{1} r_{1}^{2} B_{2}\left(\alpha_{1}\right) & q_{1} r_{1}^{2} B_{3}\left(\alpha_{1}\right) \\
q_{2} A_{0}\left(\alpha_{2}\right) & q_{2} A_{1}\left(\alpha_{2}\right) & q_{2} r_{2}^{2} A_{2}\left(\alpha_{2}\right) & q_{2} r_{2}^{2} A_{3}\left(\alpha_{2}\right) \\
q_{3} B_{0}\left(\alpha_{3}\right) & q_{3} B_{1}\left(\alpha_{3}\right) & q_{3} r_{1}^{2} B_{2}\left(\alpha_{3}\right) & q_{1} r_{1}^{2} B_{3}\left(\alpha_{3}\right) \\
\vdots & \vdots & \vdots & \vdots \\
q_{n-1} B_{0}\left(\alpha_{n-1}\right) & q_{n-1} B_{1}\left(\alpha_{n-1}\right) & q_{n-1} r_{1}^{2} B_{2}\left(\alpha_{n-1}\right) & q_{n-1} r_{1}^{2} B_{3}\left(\alpha_{n-1}\right) \\
q_{n} A_{0}\left(\alpha_{n}\right) & q_{n} A_{1}\left(\alpha_{n}\right) & q_{n} r_{2}^{2} A_{2}\left(\alpha_{n}\right) & q_{n} r_{2}^{2} A_{3}\left(\alpha_{n}\right) \\
\ldots & q_{1} r_{1}^{n-2} B_{n-2}\left(\alpha_{1}\right) & q_{1} r_{1}^{n-2} B_{n-1}\left(\alpha_{1}\right) \\
\ldots & q_{2} r_{2}^{n-2} A_{n-2}\left(\alpha_{2}\right) & q_{2} r_{2}^{n-2} A_{n-1}\left(\alpha_{2}\right) \\
\cdots & q_{3} r_{1}^{n-2} B_{n-2}\left(\alpha_{3}\right) & q_{3} r_{1}^{n-2} B_{n-1}\left(\alpha_{3}\right) \\
\cdots & \vdots & \vdots \\
\cdots & q_{n-1} r_{1}^{n-2} B_{n-2}\left(\alpha_{n-1}\right) & q_{n-1} r_{1}^{n-2} B_{n-1}\left(\alpha_{n-1}\right) \\
\cdots & q_{n} r_{2}^{n-2} A_{n-2}\left(\alpha_{n}\right) & q_{n} r_{2}^{n-2} A_{n-1}\left(\alpha_{n}\right)
\end{array}\right] .
$$

We write the $s$ th powers of the matrix $K_{n}$ as

$$
K_{n}^{s}=L_{n} J_{n}^{s} L_{n}^{-1}=W(s)=\left(w_{i j}(s)\right) .
$$

Then

$$
w_{i j}(s)=\left\{\begin{array}{lll}
0 & & \text { if } \quad(-1)^{i+j}=-1 \\
\sum_{z=1}^{\frac{n}{2}} q_{2 z-1} r_{1}^{l} \alpha_{2 z-1}^{s} B_{i-1}\left(\alpha_{2 z-1}\right) B_{j-1}\left(\alpha_{2 z-1}\right) & j=1,3, \ldots, n-1 & \\
\sum_{z=1}^{\frac{n}{2}} q_{2 z} r_{2}^{l} \alpha_{2 z}^{s} A_{i-1}\left(\alpha_{2 z}\right) A_{j-1}\left(\alpha_{2 z}\right) & j=2,4, \ldots, n & \text { if } \quad(-1)^{i+j}=1
\end{array}\right.
$$

here $r_{1}=\sqrt{\frac{b_{1}}{c_{1}}}, r_{2}=\sqrt{\frac{b_{2}}{c_{2}}}$

$$
\begin{aligned}
& l=\left\{\begin{array}{ll}
j-1, \quad j=1,3,5, \ldots, n-3, n-1 \\
j-2, \quad j=2,4,6, \ldots, n-2, n
\end{array},\right. \\
& q_{i}= \begin{cases}\frac{4-\left(\frac{\alpha_{i}-a_{1}}{\sqrt{b_{1} c_{1}}}\right)^{2}}{n+2}, & i=2 t+1(t \in \mathbb{N}) \\
\frac{4-\left(\frac{\alpha_{i}-a_{2}}{\sqrt{b_{2} c_{2}}}\right)^{2}}{n+2}, & i=2 t(t \in \mathbb{N}) .\end{cases}
\end{aligned}
$$

and $\alpha_{k}$ are the eigenvalues of the matrix $K_{n}(n=2 t, t \in \mathbb{N})$.

Corollary 8 Let $K_{n}$ be $n$-square $\left(n=2 t, t \in \mathbb{N} ; a_{1}, a_{2}, b_{1}, b_{2}, c_{1}, c_{2} \in \mathbb{C} \backslash\{0\}\right)$ pentadiagonal 2-Toeplitz matrix as in (5), from Theorem 7

$$
a_{1} \neq 2 \sqrt{b_{1} c_{1}} \cos \left(\frac{(k+1) \pi}{n+2}\right)
$$

$(k=2 t+1, t \in \mathbb{N})$ and

$$
a_{2} \neq 2 \sqrt{b_{2} c_{2}} \cos \left(\frac{k \pi}{n+2}\right) .
$$


$(k=2 t, t \in \mathbb{N})$. In that case, there exists the inverse and negative integer powers of the matrix $K_{n}$.

\section{Numerical examples}

Example 9 Taking $n=6$ in Theorem 7, we obtain

$$
\begin{aligned}
J_{6} & =\operatorname{diag}\left(\alpha_{1}, \alpha_{2}, \alpha_{3}, \alpha_{4}, \alpha_{5}, \alpha_{6}\right) \\
& =\operatorname{diag}\left(a_{1}-\sqrt{2 b_{1} c_{1}}, a_{2}-\sqrt{2 b_{2} c_{2}}, a_{1}, a_{2}, a_{1}+\sqrt{2 b_{1} c_{1}}, a_{2}+\sqrt{2 b_{2} c_{2}}\right)
\end{aligned}
$$

and

$$
\begin{aligned}
& K_{6}^{s}=L_{6} J_{6}^{s} L_{6}^{-1}=W(s) \\
&=\left(w_{i j}(s)\right)=\left[\begin{array}{cccccc}
x_{1} & 0 & x_{7} & 0 & x_{11} & 0 \\
0 & x_{2} & 0 & x_{8} & 0 & x_{12} \\
x_{3} & 0 & x_{9} & 0 & x_{7} & 0 \\
0 & x_{4} & 0 & x_{10} & 0 & x_{8} \\
x_{5} & 0 & x_{3} & 0 & x_{1} & 0 \\
0 & x_{6} & 0 & x_{4} & 0 & x_{2}
\end{array}\right], \\
& x_{1}=\frac{1}{4}\left[\left(a_{1}+\sqrt{2 b_{1} c_{1}}\right)^{s}+2 a_{1}^{s}+\left(a_{1}-\sqrt{2 b_{1} c_{1}}\right)^{s}\right] \\
& x_{2}=\frac{1}{4}\left[\left(a_{2}+\sqrt{2 b_{2} c_{2}}\right)^{s}+2 a_{2}^{s}+\left(a_{2}-\sqrt{2 b_{2} c_{2}}\right)^{s}\right] \\
& x_{3}=\frac{\sqrt{2}}{4} r_{1}^{-1}\left[\left(a_{1}+\sqrt{2 b_{1} c_{1}}\right)^{s}-\left(a_{1}-\sqrt{2 b_{1} c_{1}}\right)^{s}\right] \\
& x_{4}=\frac{\sqrt{2}}{4} r_{2}^{-1}\left[\left(a_{2}+\sqrt{2 b_{2} c_{2}}\right)^{s}-\left(a_{2}-\sqrt{2 b_{2} c_{2}}\right)^{s}\right] \\
& x_{5}= \frac{1}{4} r_{1}^{-2}\left[\left(a_{1}+\sqrt{2 b_{1} c_{1}}\right)^{s}-2 a_{1}^{s}+\left(a_{1}-\sqrt{2 b_{1} c_{1}}\right)^{s}\right] \\
& x_{6}=\frac{1}{4} r_{2}^{-2}\left[\left(a_{2}+\sqrt{2 b_{2} c_{2}}\right)^{s}-2 a_{2}^{s}+\left(a_{2}-\sqrt{2 b_{2} c_{2}}\right)^{s}\right] \\
& x_{7}=\frac{\sqrt{2}}{4} r_{1}\left[\left(a_{1}+\sqrt{2 b_{1} c_{1}}\right)^{s}-\left(a_{1}-\sqrt{2 b_{1} c_{1}}\right)^{s}\right] \\
& x_{8}=\frac{\sqrt{2}}{4} r_{2}\left[\left(a_{2}+\sqrt{2 b_{2} c_{2}}\right)^{s}-\left(a_{2}-\sqrt{2 b_{2} c_{2}}\right)^{s}\right] \\
& x_{9}=\frac{1}{2}\left[\left(a_{1}+\sqrt{2 b_{1} c_{1}}\right)^{s}+\left(a_{1}-\sqrt{2 b_{1} c_{1}}\right)^{s}\right] \\
& x_{10}=\frac{1}{2}\left[\left(a_{2}+\sqrt{2 b_{2} c_{2}}\right)^{s}+\left(a_{2}-\sqrt{2 b_{2} c_{2}}\right)^{s}\right] \\
& x_{11}=\frac{1}{4} r_{1}^{2}\left[\left(\left(a_{1}+\sqrt{2 b_{1} c_{1}}\right)^{s}-2 a_{1}^{s}+\left(a_{1}-\sqrt{2 b_{1} c_{1}}\right)^{s}\right]\right. \\
& x_{12}=\frac{1}{4} r_{2}^{2}\left[\left(a_{2}+\sqrt{2 b_{2} c_{2}}\right)^{s}-2 a_{2}^{s}+\left(a_{2}-\sqrt{2 b_{2} c_{2}}\right)^{s}\right] .
\end{aligned}
$$

Example 10 Taking $s=3, n=8, a_{1}=1, a_{2}=i+1, b_{1}=3, b_{2}=i+3, c_{1}=5$ and $c_{2}=i+5$ in Theorem 7 , we obtain

$$
\begin{array}{r}
J_{8}=\operatorname{diag}\left(\alpha_{1}, \alpha_{2}, \alpha_{3}, \alpha_{4}, \alpha_{5}, \alpha_{6}, \alpha_{7}, \alpha_{8}\right) \\
=\operatorname{diag}(-5.267,-5.280-0.668 i,-1.394,-1.399-0.363 i, \\
\quad 3.3394,3.399+1.637 i, 7.267,7.280+2.668 i)
\end{array}
$$


and

$$
\begin{aligned}
K_{8}^{3}=L_{8} J_{8}^{3} L_{8}^{-1}=W(3) & \\
=\left(w_{i j}(3)\right)= & {\left[\begin{array}{ccccc}
46 & 0 & 99 & 0 & 27 \\
0 & 16+68 i & 0 & 62+94 i & 0 \\
165 & 0 & 91 & 0 & 144 \\
0 & 118+138 i & 0 & 34+134 i & 0 \\
75 & 0 & 240 & 0 & 91 \\
0 & 42+102 i & 0 & 180+192 i & 0 \\
125 & 0 & 75 & 0 & 165 \\
0 & 110+74 i & 0 & 42+102 i & 0
\end{array}\right.}
\end{aligned}
$$

$$
\left.\begin{array}{ccc}
0 & 27 & 0 \\
6+42 i & 0 & 18+26 i \\
0 & 27 & 0 \\
96+12 i & 0 & 6+42 i \\
0 & 99 & 0 \\
34+134 i & 0 & 62+94 i \\
0 & 46 & 0 \\
118+138 i & 0 & 16+68 i
\end{array}\right] .
$$

Example 11 Taking $s=-4, n=10, a_{1}=1, a_{2}=2, b_{1}=3, b_{2}=4, c_{1}=5$ and $c_{2}=6$ in Theorem 7, we get

$$
\begin{aligned}
J_{10} & =\operatorname{diag}\left(\alpha_{1}, \alpha_{2}, \alpha_{3}, \alpha_{4}, \alpha_{5}, \alpha_{6}, \alpha_{7}, \alpha_{8}, \alpha_{9}, \alpha_{10}\right) \\
& =\operatorname{diag}(-5.7082,-6.4853,-2.8730,-2.8990,1,2,4.8730,6.8990,7.7082,10.4853)
\end{aligned}
$$

and

$$
\begin{aligned}
K_{10}^{-4}=L_{10} J_{10}^{-4} L_{10}^{-1}=W(-4) & \\
& =\left(w_{i j}(-4)\right)=\left[\begin{array}{ccccc}
0.3375 & 0 & -0.0026 & 0 & -0.1999 \\
0 & 0.0245 & 0 & -0.0029 & 0 \\
-0.0043 & 0 & 0.0044 & 0 & -0.0001 \\
0 & -0.0043 & 0 & 0.0038 & 0 \\
-0.5552 & 0 & -0.0002 & 0 & 0.3337 \\
0 & -0.0311 & 0 & -0.0002 & 0 \\
0.0067 & 0 & -0.0063 & 0 & -0.0002 \\
0 & 0.0062 & 0 & -0.0052 & 0 \\
0.9148 & 0 & 0.0067 & 0 & -0.5552 \\
0 & 0.0388 & 0 & 0.0062 & 0
\end{array}\right.
\end{aligned}
$$




$\left.\begin{array}{ccccc}0 & 0.0015 & 0 & 0.1186 & 0 \\ -0.0138 & 0 & 0.0018 & 0 & 0.0077 \\ 0 & -0.0023 & 0 & 0.0015 & 0 \\ -0.0001 & 0 & 0.0023 & 0 & 0.0018 \\ 0 & -0.0001 & 0 & -0.1999 & 0 \\ 0.0210 & 0 & -0.0001 & 0 & -0.0138 \\ 0 & 0.0044 & 0 & -0.0026 & 0 \\ -0.0001 & 0 & 0.0038 & 0 & -0.0029 \\ 0 & -0.0043 & 0 & 0.3375 & 0 \\ 0.0311 & 0 & -0.0043 & 0 & 0.0245\end{array}\right]$.

\section{References}

[1] M. J. C. Gover, The Eigenproblem of a Tridiagonal 2-Toeplitz Matrix, Linear Algebra and Its Applications, 198 (1994) 198:63-78.

[2] A. D. A. Hadj and M. Elouafi, On the characteristic polynomial, eigenvectors and determinant of a pentadiagonal matrix, Applied Mathematics and Computation, 198 (2008) 634-642.

[3] J. Rimas, Explicit expression for powers of tridiagonal 2-Toeplitz matrix of odd order, Linear Algebra and its Applications, 436 (2012) 3493-3506.

[4] M. Elouafi, On a relationship between Chebyshev polynomials and Toeplitz determinants, Applied Mathematics and Computation, 229 (2014) 27-33.

[5] R. Álvarez-Nodarse, J. Petronilho, N.R. Quintero, On some tridiagonal kToeplitz matrices: Algebraic and analytical aspects. Applications, Journal of Computational and Applied Mathematics, 184 (2005) 518-537.

[6] S. Arslan, F. Köken, D. Bozkurt, Positive integer powers and inverse for one type of even order symmetric pentadiagonal matrices, Applied Mathematics and Computation, 219 (2013) 5241-5248.

[7] A. Öteleş and M. Akbulak, Positive integer powers of certain complex tridiagonal matrices, Applied Mathematics and Computation, 219 (2013) 10448-10455.

[8] H. Wu, On Positive Integer Powers of Toeplitz Matrices, Journal of Mathematics Research, Vol. 5 No. 4 (2013) 52-57.

[9] H. K. Duru and D. Bozkurt, Integer powers of certain complex pentadiagonal Toeplitz matrices, Conference on Matrix Analysis and its ApplicationsMATTRIAD 2015, Coimbra-Portugal, 162-163.

[10] J. C. Mason and D. C. Handscomb, Chebyshev Polynomials, CRC Press, Washington, 2003.

[11] P.Horn, Ch.Johnson, Matrix Analysis, Cambridge University Press, Cambridge, 1986. 
[12] Thomas Koshy, Fibonacci and Lucas Numbers with Applications, John Wiley and Sons, NY, 2001. 